\title{
The role of near-patient coeliac serology testing in the follow-up of patients with coeliac disease
}

\author{
D A George, ${ }^{1}$ L L Hui, ${ }^{2}$ D Rattehalli, ${ }^{1}$ T Lovatt, ${ }^{1}$ I Perry, ${ }^{1}$ M Green, ${ }^{1}$ \\ K Robinson, ${ }^{1}$ J R F Walters, ${ }^{2}$ M J Brookes ${ }^{1}$
}

${ }^{1}$ Department of

Gastroenterology, New Cross

Hospital, Wolverhampton, UK

${ }^{2}$ Department of

Gastroenterology, Imperial

College London, London, UK

\section{Correspondence to}

Dr Matthew Brookes,

Department of Gastroenterology,

New Cross Hospital,

Wolverhampton Road,

Wolverhampton WV10 OQP, UK

m.j.brookes@bham.ac.uk

Received 8 May 2013

Revised 19 July 2013

Accepted 24 July 2013

Published Online First

3 September 2013
To cite: George DA, Hui $L L$, Rattehalli $\mathrm{D}$, et al. Frontline Gastroenterology 2014;5:

20-25.

\begin{abstract}
Objective This pilot study was undertaken to assess the validity and effectiveness of nearpatient coeliac immunological testing, compared to standard laboratory immunological techniques, used in the context of dietician-led coeliac disease follow-up clinics.

Design The study was designed in two phases, each assessing the near-patient test and standard laboratory immunological techniques. Phase 1 analysed stored serum samples; Phase 2 analysed whole blood from patients attending the dietician-led coeliac disease clinics.
\end{abstract}

Setting Patients were recruited from New Cross Hospital, Wolverhampton ( $n=50)$, and Imperial College London $(n=30)$, between March 2010 and February 2011.

Patients Those with a diagnosis of coeliac disease for greater than 12 months attending dietician-led coeliac disease clinics.

Interventions In addition to whole blood taken for routine analysis, patients required a capillary finger-prick blood sample.

Main outcome measure To determine if the whole blood and serum near-patient test results were in correlation with outcomes of standard laboratory evaluation.

Results Phase 1 demonstrated that the nearpatient serum test had a sensitivity of $93.5 \%(95 \%$ Cl $0.79 \%$ to $0.98 \%)$, specificity of $94.9 \%(0.83 \%$ to $0.99 \%$ ), when compared to standard laboratory ELISA. Phase 2, involving patients whole blood, had a sensitivity of $77.8 \%(0.45 \%$ to $0.93 \%)$, and specificity of $100 \%(0.94 \%$ to $1 \%)$.

Conclusions This pilot study has demonstrated that there appears to be a role for near-patient testing in coeliac disease, but further studies are recommended.

\section{INTRODUCTION}

Coeliac disease is a common autoimmune disorder precipitated by the ingestion of wheat gluten and similar proteins in barley and rye. Population studies suggest that this condition affects around $1 \%$ of Europeans. ${ }^{1}$ The disease is commonly associated with a number of autoimmune disorders through its genetic linkage with the HLA-DQ2 and -DQ8 haplotypes. ${ }^{2}$ The diagnosis can be made clinically where patients may present with a plethora of features, including lethargy, tiredness, abdominal bloating, or diarrhoea. Only a minority of patients however, will present with classical malabsorptive problems, including steatorrhoea and/or weight loss. ${ }^{3}$

The investigation of coeliac disease has been aided by the increasing use and reliability of laboratory-based serological autoantibody testing. In 1984, IgA Endomysial antibodies (EmAs) were first described directed against the intermyofibril substance of smooth muscle, suggesting a high specificity for patients with coeliac disease, and a correlation between EmAs titre and severity of small bowel histology. ${ }^{1}$ Further investigations have confirmed that EmAs were superior to serum antigiadin antibody and antireticulin antibody, previously used for diagnostic assessment of patients with symptoms consistent with coeliac disease. ${ }^{2-4}$ In 1997, Dieterich et al ${ }^{5}$ identified tissue transglutaminase (tTGA) as the antigen against which EmA was directed, and since this, tTGA testing has followed.

Near-patient testing has recently been introduced to allow for bedside testing for tTGA, using immunochromatographic assays, and these have demonstrated good efficacy when compared with serum antibody testing in the diagnosis of coeliac disease. ${ }^{67}$ The sensitivities of near-patient testing kits compared with laboratory 
ELISAs, have been reported to be between $96 \%$ and $100 \%$ with specificities of between $95 \%$ and $100 \%{ }^{6}$ When these were tested in 51 patients with untreated known coeliac disease and 36 controls they demonstrated a similar efficacy to the laboratory serum EmA and tTGA with all tests having $100 \%$ specificity. ${ }^{8}$

The ease of use of these near-patient blood droplet kits has led to an enthusiastic response for investigating potential cases of coeliac disease by paramedical teams including district nurses. ${ }^{9}$ However, further data is required to confirm its full efficacy in this setting, and there are no studies to look at the role of near-patient testing in the follow-up of treated cases of coeliac disease.

Dietician-led follow-up of patients with coeliac disease now involves a full dietary history and serological assessment to determine a patient's compliance with a gluten-free diet (GFD). The subsequent laboratory-based immunological testing can then take up to 1 week to be made available (any elevation in serum antibody levels signifies a patient's noncompliance). This delay in the availability of laboratorybased assays often means that a patient's dietary assessment in clinic is 'blind' to their immunology, which is inefficient and can waste the patient's and the dietician's time. In the current clinical setting, if the laboratory immunology is positive, the dietician often has to make a further follow-up appointment to reassess the GFD.

If the dietician had access to a simple, efficient and reliable near-patient immunological test, the assessment of the patient's GFD could be more thorough, and the patient could be informed of their results at the same clinic. Although this technology is available and has been investigated in the diagnosis of coeliac disease, it has not yet been evaluated in this setting.

The aim of this pilot study is to assess the validity and effectiveness of near-patient immunological testing used in the context of dietician-led coeliac disease clinics, compared to standard laboratory immunological evaluation. Our hypothesis is that the results of the near-patient testing will be similar to the outcomes of standard laboratory evaluation, in the follow-up of patients with coeliac disease.

\section{METHODS}

The study was undertaken in two phases: (1) laboratory stored serum samples and (2) patients who were recruited from the dietician-led coeliac disease outpatient clinics.

\section{Patients}

Patients were identified at Imperial College London, between 2 March and 11 May 2010, and at New Cross Hospital, Wolverhampton, between 14 September 2010 and 1 February 2011. Patients were screened and recruited from the dietician-led coeliac disease outpatient clinics. The group involved patients over the age of 16 years with an established histological diagnosis of coeliac disease, based on the modified Marsh criteria for the grading of villus atrophy, ${ }^{10}$ who had received treatment for at least 12 months. Those patients fulfilling the inclusion and exclusion criteria (age <16 years, pregnant, known existing liver disease (liver fibrosis or above), rheumatoid arthritis, psoriatic arthritis, ankylosing spondylitis, end-stage heart failure) were included.

In this prospective evaluation, 80 consecutive patients (median age 58 years, range 18-89) with previously diagnosed biopsy-proven coeliac disease and known serum total IgA levels took part. The time since diagnosis, and presumed GFD was from 12 months to 50 years (median: 2.17 years).

\section{Clinical data collection}

Data obtained during the consultation was a dietary evaluation, including whether a patient's GFD is assumed compliant or non-compliant (if patient openly/ obviously not compliant, has symptoms, or is known from past medical history), current and previous symptoms, known complications of coeliac disease, and body mass index. As per normal protocol, patients who were deemed symptomatic, non-compliant or had positive laboratory serology were referred for further endoscopic and histological assessment.

\section{Near-patient testing}

Medical personnel trained in performing the nearpatient test included research nurses, dieticians and a medical student. After receiving the patient's consent, a capillary finger prick (whole) blood sample was used to test for tTGA (IgG and IgA) using the 'Biohit Coeliac Quick Test' near-patient kit as per manufacturer's instructions. This test detects IgA, IgG and IgM classes of antibodies against tTGA, and is specific for whole blood requiring no laboratory expertise in its execution. All reagents were available within the single-use kit. The result of this test was read on site and available within $10 \mathrm{~min}$. A positive test led to further dietary assessments. The positivity can be further divided into a positive or strong positive result, depending upon the intensity of near-patient test indicator. In order to minimise interobserver variation, two independent researchers, in a blinded fashion, read all near-patient testing samples from the patient cohort.

\section{Blood samples}

During Phase 1, stored serum samples were analysed using the near-patient test, and analysed for antibody levels using standard ELISA techniques.

In addition to the capillary finger prick, blood samples taken from patients in Phase $2,20 \mathrm{~mL}$ of whole blood was drawn and analysed for full blood count, serum liver function, calcium profile, immunoglobulins vitamin B12, folate and ferritin, using standard laboratory techniques. 


\section{Serum antibody measurements}

The IgA class serum antibodies against TG2 were measured with human recombinant TG2 using the Celikey (Pharmacia Diagnostics, Freiburg, Germany) ELISA, according to the manufacturer's instructions. Cut-off for positivity was $5 \mathrm{U} / \mathrm{mL}$. EMA was determined on monkey oesophagus sections by indirect immunofluorescence as described elsewhere. ${ }^{11}$ Samples reactive at a serum dilution of 1:2.5 were considered positive.

\section{Statistics}

Data from each phase was analysed to assess the sensitivity and specificity (Phase 1 stored serum samples; Phase 2 whole blood samples) between the laboratorybased ELISA techniques and the near-patient test using Fishers Exact test using SPSS (V.16) for Windows. Positive and negative predictive values were determined for the respective assays.

\section{RESULTS}

\section{Serum ELISA and near-patient testing (Phase 1)}

Phase 1 of the study analysed a total of 70 serum samples. The established laboratory ELISA techniques identified 31 samples (44.3\%) to be positive for IgA tTGA, IgG tTGA, EmA or immunoglobulin levels. The near-patient test similarly identified 31 samples to be positive for elevated levels of tTGA (table 1). Two false negative near-patient tests were seen in patients with low tTGA titres $(15$ and $8 \mathrm{U} / \mathrm{mL})$. These cases were not shown to have potential confounding diagnoses, such as raised IgA, liver disease or abnormal biopsy findings.

\section{Whole blood ELISA and near-patient testing (Phase 2)}

Phase 2 prospectively identified 50 patients at the New Cross Hospital and 30 patients from Imperial College London $(\mathrm{n}=80)$. The majority of the patients were women $(72.5 \%)$, the mean age of 57.4 years (SD 14.52) with a mean age at diagnosis of 42.9 years (SD 19.3).

Of the near-patient test true positive results $(n=7)$, stronger positives accounted for higher levels of laboratory tTGA levels $(53,59 \mathrm{U} / \mathrm{mL}$, and three $>80 \mathrm{U} / \mathrm{mL}$ ), whereas, weaker positives accounted for lower levels $(20 \mathrm{U} / \mathrm{mL}$, and $50 \mathrm{U} / \mathrm{mL})$. However, false near-patient test negative results $(n=2)$ were found in patients whose laboratory levels were $20 \mathrm{U} / \mathrm{mL}$ and $35 \mathrm{U} / \mathrm{mL}$.

\section{Further details on patients recruited from New Cross Hospital only}

The majority of patients were women (74.0\%), the mean age was 55 years, a mean age at diagnosis of 46.4 years with the mean time since diagnosis was 9.17 years (median 4.5 years). Prior to the blood test results, only 35 patients $(70 \%)$ were assumed compliant with their GFD, whereas the laboratory findings confirmed that 46 patients (92.0) were compliant.

A single false negative result was seen in a patient with a high tTGA titre of $79.0 \mathrm{U} / \mathrm{mL}$. Three patients were true positives with tTGA titres of $50.0 \mathrm{U} / \mathrm{mL}$ and $>80.0 \mathrm{U} / \mathrm{mL}$ in two patients. One of these cases was assumed to have been compliant with a GFD at the clinic assessment. The mean age of these true positive patients was 44 years and they had been diagnosed with coeliac disease for an average of 16 months. At their most recent consultation, the symptoms were mainly of fatigue $(66.6 \%)$ and bone/ joint pains (66.6\%). Blood levels showed a low level of haemoglobin in one patient $(9.6 \mathrm{~g} / \mathrm{dL})$, and ferritin in two $(3.9 \mathrm{ng} / \mathrm{mL}$ and $14.3 \mathrm{ng} / \mathrm{mL})$, with normal ranges for platelets, White Cell Count (WCC), Mean Corpuscular Volume (MCV), vitamin B12 and folate levels.

Forty-six patients (92\%) were true negatives with tTGA levels ranging from $0.1 \mathrm{U} / \mathrm{mL}$ to $30.0 \mathrm{U} / \mathrm{mL}$ (table 2). The mean age was 57 years, mean age at diagnosis 47.3 years, and have had coeliac disease for an average of 9.6 years.

The majority of patients were assumed compliant (34; 68\%), eight assumed non-compliant, four however, were unknown. The main prediagnostic symptoms of this group were abdominal bloating, abdominal pain and fatigue (table 3 ).

Table 1 Statistical significance of near-patient and corresponding laboratory test results, for stored serum and whole blood samples

\begin{tabular}{|c|c|c|c|c|c|}
\hline & \multicolumn{5}{|l|}{ Laboratory testing } \\
\hline & \multicolumn{3}{|c|}{ Stored serum samples (Phase 1) } & \multicolumn{2}{|c|}{ Whole blood samples (Phase 2) } \\
\hline & \multirow[b]{2}{*}{ ELISA tTGA $(n=70)$} & \multirow[b]{2}{*}{$\lg A$ tTGA $(n=50)$} & \multirow[b]{2}{*}{ EMA $(n=50)$} & \multicolumn{2}{|l|}{ ELISA tTG } \\
\hline & & & & Both sites $(n=80)$ & New Cross Hospital $(n=50)$ \\
\hline Sensitivity (\%) & 93.5 & 92.6 & 93.5 & 77.8 & 75.0 \\
\hline Specificity (\%) & 94.9 & 95.7 & 94.7 & 100 & 100 \\
\hline PPV (\%) & 93.5 & 96.2 & 96.7 & 100 & 100 \\
\hline NPV $(\%)$ & 94.9 & 91.7 & 90 & 97.3 & 97.9 \\
\hline p Value & $<0.05$ & $<0.05$ & $<0.05$ & $<0.05$ & $<0.01$ \\
\hline
\end{tabular}

$p$ Value: statistically significant if $p<0.05$.

EMA, endomysial antibody; IgA tTG, tissue transglutaminase antibody; NPV, negative predictive value; PPV, positive predictive value. 
Table 2 Levels of tTGA in true negative results $(n=46)$

\begin{tabular}{lll}
\hline tTGA $(\mathrm{U} / \mathrm{mL})$ & Frequency $(\mathrm{n})$ & Percentage \\
\hline$<1.0$ & 17 & 37.0 \\
$\geq 1.0-<2.0$ & 6 & 13.0 \\
$\geq 2.0-<4.0$ & 7 & 15.2 \\
$\geq 4.0-<8.0$ & 9 & 19.6 \\
$\geq 8.0$ & 7 & 15.2 \\
\hline
\end{tabular}

Blood results for each patient presenting to New Cross Hospital were available within 1 week of the consultation. The majority of the blood levels were within the normal range, however, $26.1 \%$ of true negatives had deficiencies in ferritin and $2.2 \%$ of folate (table 4).

All available data on patients recruited from Imperial College London are shown in table 1.

\section{DISCUSSION}

We have demonstrated the high sensitivity and specificity of a near-patient test with an efficacy that is comparable to established laboratory immunology, using the patients' whole blood and stored serum in the follow-up of patients with coeliac disease.

Current management of patients with established diagnosis of coeliac disease, highlights the importance of adherence to the GFD and of regular dietetics assessment. Compliance with the GFD involves a dietary history and the measurement of serum laboratory antibodies, but a repeat duodenal biopsy is not routinely advocated. ${ }^{12} 13$ Indeed, it is now commonplace for coeliac disease patients on a GFD with a complete resolution of symptoms and negative follow-up laboratory tTGA to not require any further histological assessment, though practice is varied due to a lack of evidence.

Given that histological surveillance is becoming less commonplace, this will emphasise the importance of accurate dietary and serological assessment follow-up of coeliac disease. ${ }^{14}$ Laboratory serology used to monitor dietary compliance is of no use in assessing a patient's compliance in the immediate clinical setting as results are often delayed.

Use of the near-patient test in the dietician-led clinics was neither invasive nor inconvenient for the patient, allowing real-time assessment of $\operatorname{IgA}, \operatorname{IgG}$ and IgM classes of antibodies against tTG. The 'Biohit Coeliac Quick Test' near-patient kit is simple to use, requiring minimal training in its use and recognition of a positive or negative reading. During the study, a second opinion was always sought if a practitioner was unclear of the reading. This can be translated to the established dietician clinic, as a practitioner taking bloods is present for confirmation. By comparison, laboratory readings are calibrated to factory settings, the near-patient test is dependent upon the practitioner's eyesight and recognition of a positive reading.

A patient was assumed compliant to their GFD by the dietician, based upon their clinical experience, patient's current symptoms and previous encounters with the patient. The dieticians state that the immediate availability of the test helped to direct their consultation in only $20 \%$ of the time. However, our findings have shown that many patients thought to have been non-compliant were in fact confirmed compliant by the near-patient test and laboratory findings. Therefore, the availability of immediate tTGA levels, and therefore confirmation of compliance, would prevent unnecessary discussions relating to diet and possible further interventions that may be needed.

The near-patient test was further analysed against the stored serum of patients with known liver cirrhosis and myeloma, without a history of coeliac disease. The IgA levels raised in both of these conditions did not lead to (false) positive results. These findings suggest that the specificity of the test is not affected by polyclonally raised IgA. Further analysis would be needed to evaluate the sensitivity of this test in coeliac patients with IgA deficiency.

A strong, positive, near-patient test result is related to higher levels of tTGA, as well as higher rates of

Table 3 Prediagnostic and current symptoms in true negative patients presenting at New Cross Hospital $(n=46)$

\begin{tabular}{|c|c|c|c|c|}
\hline \multirow{2}{*}{$\frac{\text { Symptoms }}{\text { Abdominal bloating }}$} & \multicolumn{2}{|c|}{ Prediagnostic (n, \%) } & \multicolumn{2}{|c|}{ Current symptoms (n, \%) } \\
\hline & 27 & 58.7 & 13 & 28.3 \\
\hline Abdominal pain & 25 & 54.3 & 8 & 17.4 \\
\hline Chronic diarrhoea & 20 & 43.5 & 3 & 6.5 \\
\hline Vomiting & 2 & 4.3 & 1 & 2.2 \\
\hline Constipation & 12 & 26.1 & 11 & 23.9 \\
\hline Pale, foul-smelling, or fatty stool & 21 & 45.7 & 6 & 13.0 \\
\hline Weight loss & 19 & 41.3 & 4 & 8.7 \\
\hline Fatigue & 29 & 63.0 & 14 & 30.4 \\
\hline Bone or joint pain & 9 & 19.6 & 18 & 39.1 \\
\hline Arthritis & 4 & 8.7 & 5 & 10.9 \\
\hline Depression or anxiety & 14 & 30.4 & 7 & 15.2 \\
\hline Other & 6 & 13.0 & 0 & - \\
\hline
\end{tabular}


Table 4 Blood levels for true negatives and true positives presenting to New Cross Hospital

\begin{tabular}{|c|c|c|c|c|c|c|c|}
\hline \multirow{3}{*}{$\begin{array}{l}\text { Values } \\
\mathrm{Hb}(\mathrm{g} / \mathrm{dL})\end{array}$} & \multirow{3}{*}{$\begin{array}{l}\text { Normal range } \\
13-18 \text { (male) } \\
11.5-16 \text { (female) }\end{array}$} & \multicolumn{3}{|c|}{ True negatives $(n=46)$} & \multicolumn{3}{|c|}{ True positives $(n=3)$} \\
\hline & & \multicolumn{2}{|c|}{$\begin{array}{l}\text { Below range } \\
(n, \%)\end{array}$} & \multirow{2}{*}{$\begin{array}{l}\text { Mean } \\
13.8\end{array}$} & \multicolumn{2}{|c|}{$\begin{array}{l}\text { Below range } \\
(\mathrm{n}, \%)\end{array}$} & \multirow{2}{*}{$\begin{array}{r}\text { Mean } \\
13.26\end{array}$} \\
\hline & & 0 & - & & 1 & 33.3 & \\
\hline Plt $\left(\times 10^{9} / \mathrm{L}\right)$ & $150-450$ & 0 & - & 294 & 0 & - & 338 \\
\hline WCC $\left(\times 10^{9} / \mathrm{L}\right)$ & $4-11$ & 2 & 4.3 & 5.9 & 0 & - & 6.77 \\
\hline$M C V(f L)$ & $80-100$ & 1 & 2.2 & 89.35 & 0 & - & 79.3 \\
\hline Vitamin B12 (ng/L) & $>150$ & 0 & - & 499.5 & 0 & - & 599.3 \\
\hline Folate $(\mu \mathrm{g} / \mathrm{L})$ & $3-30$ & 1 & 2.2 & 9.03 & 0 & - & 13.28 \\
\hline Ferritin $(\mu \mathrm{g} / \mathrm{L})$ & $28-365$ & 12 & 26.1 & 65.1 & 2 & 66.7 & 17.17 \\
\hline
\end{tabular}

$\mathrm{Hb}$, haemoglobin; Plt, platelet; WCC, White Cell Count; MCV, Mean Corpuscular Volume.

symptoms and deficiencies, such as haemoglobin and ferritin (66.7\%). However, it was shown that a large proportion of true negative patients, despite having been compliant with their GFD, were still deficient in ferritin $(26.1 \%)$ and remained symptomatic of coeliac disease (although the frequency of symptoms had halved since their initial diagnosis). This may reflect an irregularity in the correlation between IgA levels, compliance with GFD, and subsequent blood deficiencies.

Unfortunately in this study, we did not examine patient serum with the near-patient test in Phase 2. It is likely that the small differences in the sensitivity and specificity seen between Phases 1 and 2 are occurring as a result of the higher concentrations of antibody present in serum rather than whole blood. In this study, it does obviously lead to differences in the statistical results, however, in wider practice, this difference is likely to be irrelevant, as the near-patient testing will only be based on whole blood sampling taken within the clinic environment. Furthermore, this project is a small pilot study and, as such, the low number of tTG positivity may have significantly influenced the statistical outcomes of this work.

Despite these shortcomings, we have demonstrated encouraging findings, and would use the results to inform the design of large controlled trials to determine the cost effectiveness of replacing current dietician-led clinics and laboratory immunological assessments with a 'one-stop' clinic assessment using the near-patient test. This will allow for a more streamlined, efficient and cost-effective clinic to be run. It would also improve our service to patients, allowing them to receive their immunology results immediately, and preventing those with positive results having to be brought back to the clinic another time to re-evaluate their diet. As this is such a common disorder, it will have a large impact on practice, reducing both the need for further clinic assessment, and reducing the need for laboratory blood samples, thus, improving the patient experience and reducing clinical costs as well.

\section{What is already known on this topic}

Near-patient immunological testing has recently been introduced to allow for bedside testing for tTGA levels in the diagnosis of coeliac disease. Results have shown a high sensitivity (96-100\%) and specificity $(95-100 \%)$ compared to standard laboratory immunological evaluation.

\section{What this study adds}

This study assesses the validity and effectiveness of near-patient testing, in the context of a dietician-led follow up clinic of treated cases of coeliac disease. Utilising the near-patient test on stored serum, there was a sensitivity of $93.5 \%$ and specificity of $94.9 \%$, when compared to standard laboratory immunological evaluation. In the clinic, patient's whole blood was shown to have a sensitivity of $77.8 \%$, and specificity of $100 \%$.

\section{How might it impact on clinical practice in the fore-} seeable future

We would utilise these encouraging results to inform the design of large controlled trials, to determine the cost-effectiveness of replacing current dietician-led clinics and laboratory immunological assessments, with a "one-stop" clinic assessment. The ultimate aim would be to enable a streamlined, efficient and cost-effective clinic to be run, and improve our service to patients by allowing them to receive their immunology results immediately, and reduce the need for laboratory blood samples. 
Acknowledgements We would like to thank Core and Dr Falk Pharma for their kind bursary, which helped fund the study.

Contributors All authors have made substantial contributions to all of the following: the conception and design of the study, or acquisition of data, or analysis and interpretation of data; drafting the article or revising it critically for important intellectual content, and final approval of the version to be submitted.

Funding $£ 2250$ bursary awarded from Dr Falk Pharma and Core.

Competing interests None.

Patient consent Obtained.

Ethics approval Obtained from Birmingham Black Country Research Ethics Committee (REC); 10/H1202/46.

Provenance and peer review Not commissioned; externally peer reviewed.

\section{REFERENCES}

1 West J, Logan RFA, Hill PG, et al. Seroprevalence, correlates, and characteristics of undetected coeliac disease in England. Gut 2003;52:960-5.

2 Sollid LM, Thorsby E. HLA susceptibility genes in celiac disease: genetic mapping and role in pathogenesis. Gastroenterology 1993;105:910-22.

3 Ferreira M, Davies S, Butlet M, et al. Endomysial antibody: is it the best screening test for celiac disease? Gut 1992:33;1633-7.

4 Lerner A, Kumar V, Iancu T. Immunological diagnosis of childhood celiac disease: comparison between antigliadin, antireticulin and antiendomysial antibodies. Clin Exp Immune 1994:95;78-82.

5 Dieterich W, Ehnis T, Bauer M, et al. Identification of tissue transglutaminase as the autoantigen of coeliac disease. Nat Med 1997;3:797-801.
6 Nemec G, Ventura A, Stefano M, et al. Looking for celiac disease: diagnostic accuracy of two rapid commercial assays. Am J Gastroent 2006:101;1597-600.

7 Korponay-Szabo IR, Raivio T, Laurila K, et al. Coeliac disease case finding and diet monitoring by point-of-care testing. Aliment Pharmacol Ther 2005:22;729-37.

8 Raivio T, Korponay-Szabo I, Collin P, et al. Performance of new rapid whole blood celiac test in adult patients with low prevalence of anti-endomysial antibodies. Dig Liver Dis 2007:39;1057-63.

9 Korponay-Szabo I, Szabados K, Pusztai J, et al. Population screening for celiac screening in primary care by district nurses using a rapid antibody test: diagnostic accuracy and feasibility study. BMJ 2007:335;1244-7.

10 Oberhuber G, Granditsch G, Vogelsang H. The histopathology of coeliac disease: time for a standardized report scheme for pathologists. Eur J Gastroenterol Hepatol 1999;11:1185-94.

11 Picarelli A, Sabbatella L, Di Tola M, et al. Nuclear fluorescence serum reactivity on monkey oesophagus: a new antibody for the follow-up of coeliac disease? Clin Exp Immunol 2010;161:417-25

12 Rostom A, Murray JA, Kagnoff MF. American Gastroenterological Association (AGA). Institute Technical Review on the Diagnosis and Management of Celiac Disease. Gastroenterology 2006;131;1981-2002.

13 Ciclitira PJ, Dewar DH, McLaughlin SD, et al. BSG guidance on coeliac disease. The management of adults with coeliac disease. London: British Society of Gastroenterology (BSG), 2010.

14 Sugai E, Nachman F, Váquez H, et al. Dynamics of celiac disease-specific serology after initiation of a gluten-free diet and use in the assessment of compliance with treatment. Dig Liver Dis 2009;42;352-8. 